'Sección de Nefrología, Servicio de Medicina Interna, Hospital del Salvador. Santiago, Chile. 2Departamento de Medicina, Facultad de Medicina,

Universidad de Chile. Santiago, Chile.

Recibido el 29 de abril de 2015, aceptado el 9 de octubre de 2015.

Correspondencia a: Dr. Ricardo Valjalo Servicio de Medicina Interna, Hospital del Salvador. Avenida Salvador 364,

Providencia. Santiago, Chile. Teléfono: (2) 25753532 rvaljalo@hotmail.com

\section{Resultados a largo plazo en trasplante renal de donantes con criterios expandidos}

\section{RICARDO VALJALO ${ }^{1,2}$, ENRIQUE REYNOLDS ${ }^{1,2}$, PATRICIA HERRERA ${ }^{1,2}$, ÓSCAR ESPINOZA ${ }^{1,2}$, FERNANDO GONZÁLEZ ${ }^{1,2}$ \\ Long-term outcomes with expanded criteria donors in kidney transplantation}

Background: Kidney transplantation of expanded criteria deceased donors (DCE) has become a common clinical practice. However, DCE outcomes are inferior compared to kidney transplants from standard criteria donors (DCS). Aim: To evaluate intermediate and long-term outcomes of DCE transplanted patients. Material and Methods: Cadaveric kidney transplants were evaluated using a retrospective cohort of eight consecutive years. Complications and longterm function of the transplant were assessed in DCE and DCS kidney recipients. Results: Of 213 patients analyzed, 34 (16\%) underwent DCE transplantation. $D C S$ recipients spent more time on the waiting list for transplantation ( $p=$ $0.04)$. DCE recipients showed higher frequency of surgical complications $(p=$ 0.04 ), vascular complications ( $p=0.02)$, acute transplant rejection $(p=0.05)$, and hospitalizations $(p=0.01)$. Creatinine $(\mathrm{mg} / \mathrm{dL})$ in DCE and DCS recipients was 2.3 and 1.5 respectively at year one $(p<0.01)$ and 2.6 and 1.6 respectively at year five $(p<0.01)$. Graft survival in the DCE group was significantly lower at 5 years (61 and 89\% respectively, $p<0.01$ ). Conclusions: DCE grafts are associated with lower survival, higher hospitalization rate and commonly develop surgical complications and rejections.

(Rev Med Chile 2016; 144: 22-29)

Key words: Aged; Donor selection; Graft rejection; Graft survival; Kidney transplantation.
$\mathrm{E}$ 1 donante cadavérico ideal es considerado un individuo joven, previamente sano, que fallece producto de una lesión traumática del encéfalo, manteniendo intacta la funcionalidad de órganos torácicos y abdominales. El aumento en la prevalencia de la enfermedad renal crónica avanzada ha provocado, a nivel mundial, un crecimiento sustancial en las listas de espera para trasplante renal cadavérico, que supera por mucho el número de riñones procurados. Esta crisis global en el suministro de órganos ha llevado a maximizar el uso de órganos obtenidos de donantes fallecidos, es así como en la actualidad donantes añosos y con condiciones antiguamente consideradas no aptas para la donación, están siendo aceptados. Estos donantes "no ideales" son actualmente denominados donantes con criterios expandidos (DCE) y se caracterizan por poseer órganos con menor reserva funcional y capacidad regenerativa, lo que se ha vinculado, posterior al trasplante, con menor sobrevida a largo plazo y con mayor probabilidad de insuficiencia primaria, función retardada del injerto y episodios de rechazo agudo, cuando son comparados con órganos de donantes con criterios estándar (DCS) ${ }^{1}$. Múltiples factores han sido implicados en estos resultados, incluyendo una masa nefronal reducida, presencia de daño renal previo, menor capacidad tisular 
regenerativa, mayor susceptibilidad a la injuria isquemia-reperfusión y elevada inmunogenicidad del injerto ${ }^{2,3}$.

Si bien los riñones provenientes de DCE presentan una menor sobrevida a largo plazo que los provenientes de DCS, Ojo y cols. demostraron que aun así, los receptores de DCE se benefician en términos de sobrevida, cuando son comparados a los pacientes en diálisis que se encuentran en lista de espera y que no logran ser trasplantados. A pesar de esto, la calidad limitada de dichos órganos hace que sigan siendo descartados con relativa frecuencia y muchos centros de trasplante prefieren no hacer uso de ellos ${ }^{5}$. Nuestro objetivo fue evaluar los resultados a mediano y largo plazo de los receptores sometidos a trasplante renal con DCE, en nuestro centro.

\section{Pacientes y Método}

Se llevó a cabo un estudio de cohorte no concurrente, considerando todos los pacientes mayores de 18 años sometidos a trasplante renal exclusivo de donante cadavérico efectuados en el Hospital del Salvador (Santiago, Chile), entre el 01 de enero de 2005 y el 31 de diciembre de 2012. Fueron considerados como DCE los donantes que cumplían los siguientes criterios sugeridos por la United Network for Organ Sharing (UNOS): Mayores de 60 años, o los fallecidos de entre 50 y 59 años con al menos 2 de las siguientes condiciones médicas: Historia de hipertensión arterial (HTA), accidente cerebrovascular como causa de defunción o creatinina sérica previa a la procura mayor a $1,5 \mathrm{mg} / \mathrm{dL}^{6}$.

Fueron considerados como DCS todos los donantes que no reuniesen los requisitos de DCE. El criterio de asignación de receptores para los DCE fue "old for old", asignándoseles por tanto, a receptores de una edad similar? ${ }^{7}$.

Todos los pacientes fueron instruidos acerca de las expectativas, riesgos y beneficios relacionados al trasplante, y firmaron consentimiento informado. En algunas circunstancias, y en caso de no existir un receptor para el segundo riñón donado, los receptores de DCE recibieron un doble trasplante renal. Los riñones donados fueron conservados hasta el trasplante utilizando soluciones de preservación. El uso de inducción con anticuerpos se reservó para los pacientes con reactividad de anticuerpos al panel de linfocitos (PRA) mayor a 20\%, y el esquema inmunosupresor inicial constó de terapia triasociada con glucocorticoides, ciclosporina y azatioprina en los pacientes con bajo nivel de sensibilización previa al trasplante y de glucocorticoides, tacrolimus y micofenolato en los pacientes con mayores niveles de sensibilización. Se utilizó ketoconazol como fármaco ahorrador de anticalcineurínicos.

Durante el seguimiento evaluamos la ocurrencia de rechazo agudo, disfunción primaria del trasplante, retraso de función del injerto (RFI), hospitalizaciones, complicaciones vasculares post-trasplante y complicaciones urológicas que necesitaron de re-intervención quirúrgica. Además, fueron evaluados a largo plazo la función renal, sobrevida del injerto y sobrevida de los receptores. Se consideró como RFI a la necesidad de diálisis durante la primera semana posterior al trasplante. La ausencia permanente de función renal posterior al trasplante, independientemente del motivo, fue considerada como disfunción primaria del trasplante. Se consideraron complicaciones quirúrgicas a la ocurrencia de complicaciones post-operatorias que requirieron de re-intervención quirúrgica (linfoceles, fístulas urinarias, colecciones, infección de herida operatoria, etc.). Fueron incluidas como complicaciones vasculares la ocurrencia de trombosis arterial o venosa del injerto, el sangrado post-operatorio desde el pedículo o a la aparición posterior de estenosis en la arteria renal del injerto. Todos los rechazos fueron confirmados histológicamente, de acuerdo a la clasificación de Banff vigente en el período en que se realizó la biopsia y tratados según el tipo de rechazo demostrado. Se contabilizaron las hospitalizaciones por cualquier motivo ocurridas durante el período de seguimiento, considerándose la razón entre las hospitalizaciones y los años de seguimiento. La velocidad de filtración glomerular (VFG) fue calculada en base a la fórmula MDRD-2. Las variables categóricas fueron evaluadas usando el test exacto de Fisher y las variables continuas utilizando la prueba t de Student, ANCOVA y U de Mann-Whitney. Utilizando curvas de KaplanMeier y análisis comparativo con log-rank, fueron analizadas las curvas de sobrevida de pacientes y de injertos, esta última censurada por muerte con injerto funcionante. Se utilizó el software estadístico SPSS v22. 


\section{Resultados}

En los ocho años fueron efectuados 215 trasplantes con donante cadavérico. Se excluyó a dos trasplantados, uno, receptor de DCS, por continuar seguimiento en otro centro $y$, el otro, por extravío de datos $(n=213)$. De estos 213 pacientes, 34 pacientes $(16 \%)$ recibieron trasplante de DCE y $179(84 \%)$ de DCS. El $47 \%$ de trasplantes de DCE fueron efectuados en los últimos 2 años del período considerado. El promedio de seguimiento fue de 4,5 años. Las características de los donantes, receptores y del trasplante están resumidas en la Tabla 1. Como era de esperarse por su definición, los DCE presentaron mayor edad (61 vs 38 años, $\mathrm{p}<0,01)$, mayor prevalencia de HTA $(\mathrm{p}<0,01)$ $\mathrm{y}$ fallecieron con mayor frecuencia por ACV $(\mathrm{p}<$ $0,01)$ que los DCS. El tiempo de espera en lista de trasplante fue significativamente menor en los receptores de DCE $(p=0,04)$. Los receptores de DCE tuvieron mayor edad (58 vs 44 años, $\mathrm{p}<0,01$ ) y discordancias HLA que el grupo de comparación.

Tabla 1. Características de donantes, receptores y del trasplante

\begin{tabular}{|c|c|c|c|}
\hline & DCE $(n=34)$ & $\operatorname{DCS}(n=179)$ & $\mathbf{p}$ \\
\hline Edad del donante (años) & $61,0 \pm 5,7$ & $38,3 \pm 12,3$ & $<0,01$ \\
\hline Muerte del donante: ACV & $19(56 \%)$ & $36(20 \%)$ & $<0,01$ \\
\hline Donante: HTA & $20(59 \%)$ & $19(11 \%)$ & $<0,01$ \\
\hline Donante: DM-2 & $1 \quad(3 \%)$ & $4(2 \%)$ & 0,59 \\
\hline Creatinina del donante (mg/dL) & $0,86 \pm 0,26$ & $0,89 \pm 0,36$ & 0,85 \\
\hline Tiempo de isquemia fría (h) & $16,6 \pm 4,1$ & $20,5 \pm 6,4$ & $<0,01$ \\
\hline Tiempo de isquemia caliente (min) & $42,7 \pm 10,8$ & $39,7 \pm 9,9$ & 0,11 \\
\hline Edad del receptor (años) & $57,7 \pm 8,5$ & $43,6 \pm 11,6$ & $<0,01$ \\
\hline Receptor: Sexo masculino & $22(65 \%)$ & $104(58 \%)$ & 0,30 \\
\hline Tiempo en lista de espera (meses) & $32,4 \pm 28,1$ & $41,9 \pm 31,6$ & 0,04 \\
\hline Trasplante renal previo & $2(6 \%)$ & $9(5 \%)$ & 0,55 \\
\hline Tiempo en diálisis (meses) & $66,7 \pm 53,2$ & $73,6 \pm 46,4$ & 0,24 \\
\hline PRA 0\% & $15(44 \%)$ & $52(29 \%)$ & 0,08 \\
\hline PRA $>20 \%$ & $5(15 \%)$ & $55(26 \%)$ & 0,11 \\
\hline HLA-mismatch & $3,7 \pm 1,3$ & $2,7 \pm 1,2$ & $<0,01$ \\
\hline Trasplante renal doble & $7(21 \%)$ & 0 & $<0,01$ \\
\hline Receptor: HTA & $22(65 \%)$ & $114(64 \%)$ & 0,54 \\
\hline Receptor: DM-2 & $3(9 \%)$ & $11(6 \%)$ & 0,39 \\
\hline Receptor: IgG CMV (+) & $30(88 \%)$ & $162(92 \%)$ & 0,33 \\
\hline Inducción con Basiliximab & $5(15 \%)$ & $35(20 \%)$ & 0,35 \\
\hline Inducción con rATG & 1 (3\%) & $12(7 \%)$ & 0,35 \\
\hline Inmunosupresión: Ciclosporina & 33 (97\%) & $167(93 \%)$ & 0,35 \\
\hline Inmunosupresión: Tacrolimus & 1 (3\%) & $11(6 \%)$ & 0,40 \\
\hline Inmunosupresión: Azatioprina & $31(91 \%)$ & $163(91 \%)$ & 0,64 \\
\hline Inmunosupresión: Micofenolato & $3(9 \%)$ & $16(9 \%)$ & 0,64 \\
\hline
\end{tabular}

Abreviaturas. ACV: Accidente cerebrovascular; HTA: Hipertensión arterial; DM-2: Diabetes mellitus tipo 2; PRA: Reactividad de anticuerpos al panel de linfocitos; HLA: Antígeno leucocitario humano; CMV: Citomegalovirus; rATG: Timoglobulina de conejo anti-timocitos. 
No hubo diferencias significativas en cuanto a los esquemas inmunosupresores de inducción o de mantenimiento empleados.

Los receptores de DCE presentaron (Tabla 2) mayor ocurrencia de complicaciones quirúrgicas $(\mathrm{p}=0,04)$, complicaciones vasculares $(\mathrm{p}=0,02)$ y de episodios de rechazo agudo confirmados por biopsia $(p=0,05)$. La tasa observada de hospitalizaciones fue significativamente mayor $(\mathrm{p}=0,01)$. No hubo diferencias entre ambos grupos en cuanto a desarrollo de RFI, disfunción primaria del injerto, neoplasias, diabetes post-trasplante y enfermedad por citomegalovirus (CMV).

La función renal en ambos grupos evidenció diferencias a partir del primer mes post-trasplante, la que se hizo significativa desde el primer año y se mantuvo durante el período de seguimiento. Los niveles de creatinina sérica al mes, al año y a los 5 años en los receptores de DCE versus DCS fueron de 2,9 vs $2,2(\mathrm{p}=0,08) ; 2,3$ vs $1,5(\mathrm{p}<0,01)$ y 2,6 vs $1,6(\mathrm{p}<0,01) \mathrm{mg} / \mathrm{dL}$, respectivamente (Figura 1$)$. La VFG calculada en base a la fórmula MDRD registrada al mes, al año y al quinto año, fue de 33 vs 40,32 vs 51 y 29 vs $54 \mathrm{~mL} / \mathrm{min}$ en receptores de DCE y DCS respectivamente (Figura 2). El análisis de ANCOVA, considerando la creatinina y VFG al mes de trasplante como covariable, también mostró que la función renal de ambos grupos, durante los años sucesivos, fueron estadísticamente diferentes, favoreciendo a los receptores de DCS. La función renal evidenciada en el subgrupo de DCE que recibió trasplante renal doble no mostró

Tabla 2. Resultados

\begin{tabular}{|lccc|}
\hline & DCE (n = 34) & DCS (n = 179) & p \\
\hline Retraso de función del injerto & $16(47 \%)$ & $90(50 \%)$ & 0,44 \\
\hline Disfunción primaria del injerto & $4(12 \%)$ & $10(6 \%)$ & 0,17 \\
Complicación quirúrgica & $11(32 \%)$ & $30(17 \%)$ & 0,04 \\
Complicación vascular & $7(21 \%)$ & $13(7 \%)$ & 0,02 \\
Rechazo agudo en primer año & $11(32 \%)$ & $32(18 \%)$ & 0,05 \\
Diabetes post-trasplante & $3(9 \%)$ & $15(8 \%)$ & 0,57 \\
Enfermedad por CMV & $2(6 \%)$ & $12(7 \%)$ & 0,61 \\
Nefropatía por BK & $2(6 \%)$ & $4(2 \%)$ & 0,25 \\
Desarrollo de neoplasias & $1(3 \%)$ & $8(5 \%)$ & 0,56 \\
Hospitalizaciones/año & $1,5 \pm 2,1$ & $0,6 \pm 0,8$ & 0,01 \\
\hline
\end{tabular}

Abreviaturas. CMV: Citomegalovirus.

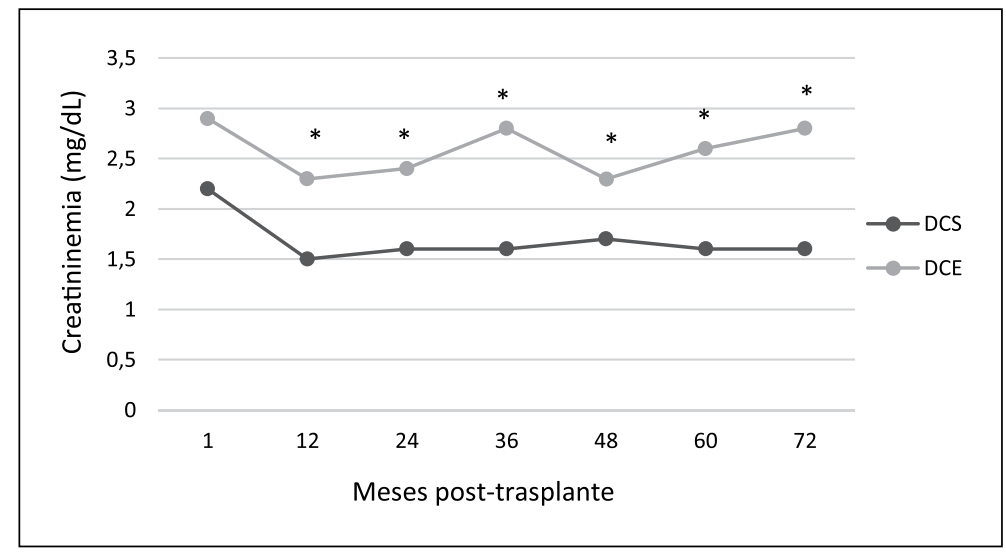

Figura 1. Creatininemia a los 6 años post-trasplante. Seguimiento de función renal medida con creatinina sérica, a los 6 años post-trasplante $\left({ }^{*} p<0,05\right)$. DCS: Donantes de criterio estándar. DCE: Donantes de criterios expandidos. 

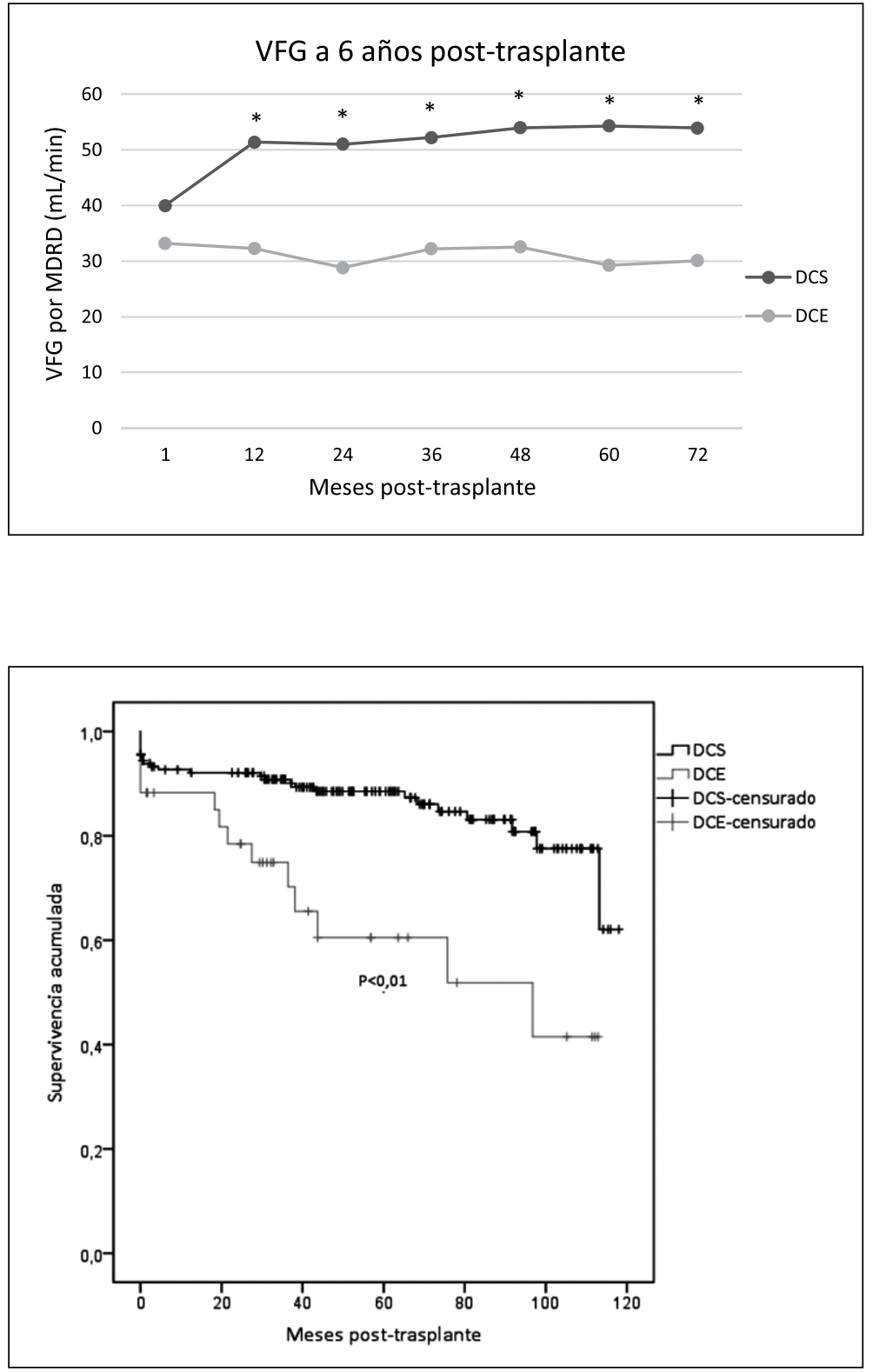

Figura 2. VFG a 6 años post-trasplante. Seguimiento de función renal con VFG calculada por fórmula MDRD, a los 6 años post-trasplante $\left({ }^{*} p<0,05\right)$. VFG: Velocidad de filtración glomerular. DCS: Donantes de criterio estándar. DCE: Donantes de criterios expandidos.

Figura 3. Tasa de supervivencia actuarial del injerto, en receptores de trasplante renal de DCE versus DCS. DCS: Donantes de criterio estándar. DCE: Donantes de criterios expandidos.

diferencias significativas, durante el período de seguimiento, respecto a los receptores de DCE que recibieron trasplante simple.

La muerte con injerto funcionante ocurrió en 4 casos (12\%) en el grupo de receptores de DCE, y en 14 casos $(8 \%)$ en el grupo de receptores de DCS $(\mathrm{p}=0,45)$. Tras censurar los casos de muerte con injerto funcionante, la tasa de supervivencia actuarial del injerto al año, 3 años y 5 años fue de $88 \%$ vs $93 \%, 75 \%$ vs $91 \%$ y 61 vs $89 \%$ en el grupo de DCE y DCS, respectivamente, siendo estas diferencias estadísticamente significativas (Figura 3).

La sobrevida de los pacientes a 5 años fue de $91 \%$ en los receptores de DCS y 74\% en los receptores de DCE, diferencia que resultó significativa (Figura 4). 


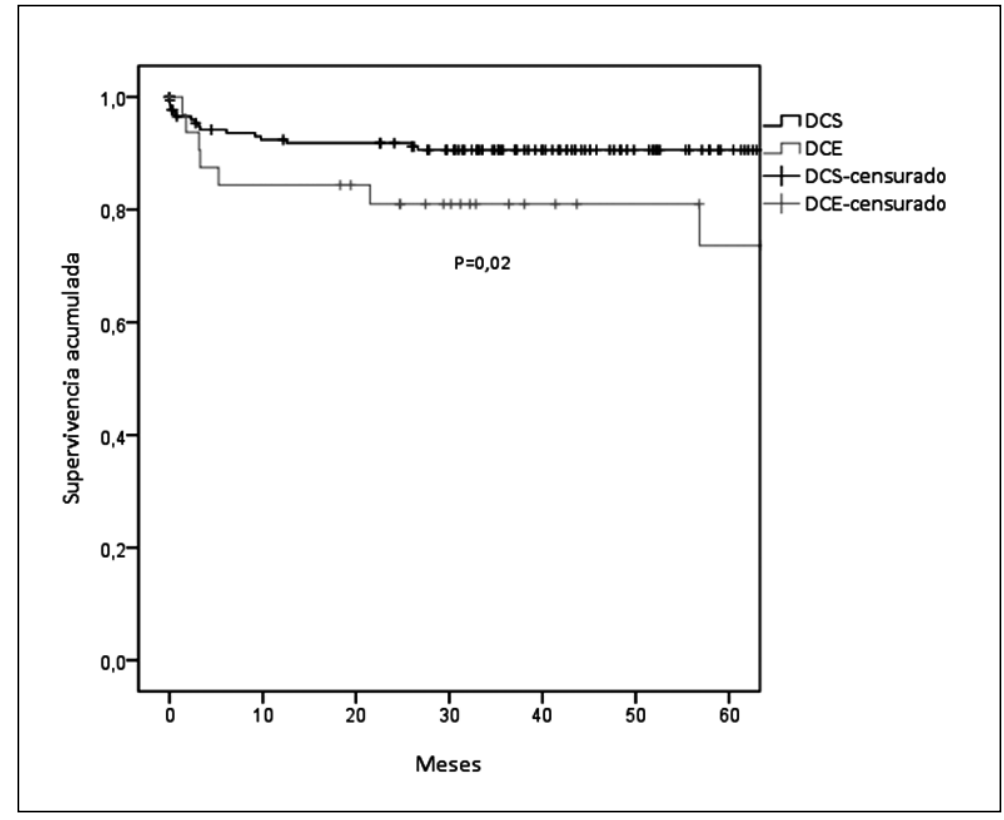

Figura 4. Tasa de supervivencia actuarial de los pacientes receptores de DCE versus DCS. DCS: Donantes de criterio estándar. DCE: Donantes de criterios expandidos.

\section{Discusión}

El aumento sostenido de la enfermedad renal crónica en Chile es responsable de que al año 2014 existan 18.160 pacientes en programa de hemodiálisis crónica, estimándose una prevalencia de 1.019 pacientes por millón de habitantes, cifra considerada elevada en comparación a países de Latinoamérica y resto del mundo ${ }^{8,9}$. Entre las diferentes modalidades de sustitución renal, el trasplante ha sido considerada la terapia de elección por brindar una mejor expectativa y calidad de vida, y por ser la opción con perfil de costo-efectividad más favorable ${ }^{10-12}$.

En nuestro país, semejante al resto del mundo, hemos observado un progresivo incremento de los pacientes en lista de espera para trasplante renal, existiendo al año 2014 una tasa de 88 por millón de habitantes, cifra marcadamente superior que la tasa de trasplantes cadavéricos a la misma fecha (12 por millón de habitantes). El problema se ve incrementado si consideramos que sólo $8,2 \%$ de pacientes en hemodiálisis crónica se encuentra en lista de espera, recuento considerado bajo con respecto a otros países, en las que esta cifra bordea el 20\% (Elgueta S. Registro nacional de trasplante renal 2014. XII Congreso latinoamericano de nefrología e hipertensión.
Santiago, Chile. 2014). Esta amplia brecha entre candidatos a trasplante y disponibilidad de órganos ha llevado a maximizar el uso de donantes vivos y cadavéricos antes considerados descartables, trasplantando a modo de ejemplo riñones de donantes con edades extremas, con diferentes morbilidades, o con incompatibilidad de grupo $\mathrm{ABO}$ o HLA. El trasplante renal con DCE ha sido cuestionado por sus peores resultados: Mayor frecuencia de disfunción primaria, RFI, rechazo agudo y menor sobrevida del injerto (aproximadamente 4 años menor) en comparación a los receptores de DCS. Tales resultados podrían ser explicados por la menor masa nefronal funcionante, mayor frecuencia de alteraciones histológicas como glomeruloesclerosis, fibrosis intersticial, atrofia tubular y arterioesclerosis, una elevada antigenicidad del injerto y una mayor susceptibilidad a nefrotoxicidad y daño inmunológico ${ }^{2,3,13}$.

En esta serie, y al igual que en la mayoría de las series publicadas, los resultados obtenidos en receptores de DCE son inferiores en términos de sobrevida del injerto, incidencia de rechazo y función renal a largo plazo. Las diferencias en función renal de ambos grupos se manifiestan precozmente desde el primer mes y se hacen significativas a partir del año en forma sostenida. Al 
aplicar un análisis de covarianza, pudimos determinar que las diferencias de creatininemia y VFG entre ambos grupos, durante el seguimiento, son independientes de la función renal obtenida al mes post-trasplante, por lo tanto, un buen resultado inicial no sería necesariamente un buen predictor de resultados satisfactorios a largo plazo. Debemos considerar que la incompatibilidad HLA en los receptores de DCE fue mayor, y el uso de inducción fue infrecuente, lo que podría haber influido en la mayor ocurrencia de eventos inmunológicos en este grupo de pacientes particularmente susceptibles. Del mismo modo, pareciere que los trasplantes de DCE no serían los candidatos más idóneos a recibir esquemas inmunosupresores libres de agentes anticalcineurínicos. La sobrevida de los receptores de DCE fue más reducida, sin embargo, no puede dejar de considerarse la gran diferencia etaria entre ambos grupos, y la probabilidad implícita de presentar con mayor frecuencia eventos desfavorables en el grupo de edad más avanzada. El tiempo en lista de espera de los receptores de DCE fue considerablemente menor, sin embargo, creemos que este tiempo es aún más bajo tras observar que muchos de los receptores de DCE ingresaron a la lista de espera de DCE tras permanecer un largo tiempo en la lista estándar.

Numerosas han sido las estrategias para mejorar los resultados de los injertos de DCE, como seleccionar receptores con bajo riesgo inmunológico y HLA compatibles, reducir los tiempos de isquemia y mejorar la criopreservación de los injertos ${ }^{14,15}$. Remuzzi y cols. han obtenidos buenos resultados utilizando criterios histológicos para la aceptación de los injertos, pudiendo de esta manera descartar o aceptar el injerto donado según la puntuación histológica obtenida; sin embargo, esta práctica requiere un gran despliegue de recursos y es irrealizable en nuestro medio ${ }^{16}$. Una serie de otras medidas han demostrado ser efectivas en optimizar la utilización de este tipo de trasplantes, sugiriéndose así llevarlos a cabo con receptores mayores de 60 años o mayores de 40 años con nefropatía diabética en los que se prevé una larga permanencia en lista de espera. En este grupo se ha mostrado una mejor sobrevida recibiendo trasplante renal de DCE que permaneciendo en hemodiálisis y lista de espera ${ }^{17,18}$.

En nuestro análisis, además, pudimos obser- var un mayor número de hospitalizaciones y de complicaciones post-quirúrgicas en los receptores de DCE. El mayor número de hospitalizaciones en este grupo fue explicado principalmente por la mayor ocurrencia de rechazos, de complicaciones quirúrgicas tardías y de infecciones respiratorias. Es importante destacar que, si bien el $21 \%$ de ellos recibió un doble trasplante renal, este subgrupo de pacientes presentó similar número de complicaciones peri-operatorias que los receptores de riñón único, sin poder demostrarse influencia de la doble intervención en la mayor ocurrencia de complicaciones quirúrgicas del grupo receptor de DCE. Son pocos los ensayos que han evaluado adecuadamente, y a largo plazo, la ocurrencia de dichos eventos en este grupo de pacientes, y los resultados reportados son dispares. Algunos autores han descrito un incremento en la frecuencia de linfoceles, mayor necesidad de re intervención quirúrgica y un riesgo aumentado de complicaciones urológicas y vasculares a corto y largo plazo ${ }^{19,20}$. Es muy probable que la alta prevalencia de arterioesclerosis, tanto del donante como del receptor, juegue un rol protagónico en la génesis de las complicaciones vasculares en los sujetos más añosos ${ }^{21}$. Además, la mayor fragilidad linfática renal, la abundancia del tejido graso perihiliar que dificulta la disección quirúrgica, y una menor vascularización a nivel ureteral, podrían ser los factores contribuyentes a la mayor ocurrencia de linfoceles y de complicaciones de la vía urinaria ${ }^{22}$.

Una de las fortalezas de nuestro estudio es el largo tiempo de seguimiento promedio $(4,5$ años), que permite evaluar los desenlaces a largo plazo; además la buena calidad y tabulación de los registros clínicos, junto a la revisión acuciosa de las fichas clínicas lograron que fueran analizados el $99 \%$ de los trasplantados en el período considerado y permitieron obtener prácticamente la totalidad de eventos ocurridos.

Finalmente, concluimos que la reducida supervivencia del injerto y mayor ocurrencia de complicaciones no significa una falta de beneficio terapéutico. Los trasplantes de DCE son una alternativa válida frente a los excesivos tiempos en lista de espera, pero deben realizarse esfuerzos adicionales y exhaustivos enfocados en seleccionar receptores adecuados, para así maximizar la utilidad del procedimiento y reducir la tasa de complicaciones. 


\section{Referencias}

1. Carter JT, Lee CM, Weinstein RJ, Lu AD, Dafoe DC, Alfrey EJ. Evaluation of the older cadaveric kidney donor: the impact of donor hypertension and creatinine clearance on graft performance and survival. Transplantation 2000; 70 (5): 765-71.

2. De Fijter J, Mallat M, Doxiadis I, Ringers J, Rosendaal F, Claas F, et al. Increased immunogenicity and cause of graft loss of old donor kidneys. J Am Soc Nephrol 2001; 12 (7): 1538-46.

3. Bajwa M, Cho YW, Pham PT, Shah T, Danovitch G, Wilkinson A, et al. Donor biopsy and kidney transplant outcomes: an analysis using the Organ Procurement and Transplantation Network/United Network for Organ Sharing (OPTN/UNOS) database. Transplantation 2007; 84 (11): 1399-405.

4. Ojo A, Hanson J, Meier-Kriesche H, Okechuwku C, Wolfe R, Leichtman A, et al. Survival in recipients of marginal cadaveric donor kidneys compared with other recipients and wait-listed transplant candidates. J Am Soc Nephrol 2001; 12 (3): 589-97.

5. Lee C, Scandling J, Pavlakis M, Markezich A, Dafoe D, Alfrey E. A review of kidneys that nobody wanted: determinants of optimal outcome. Transplantation 1998; 65 (2): 213-9.

6. Rosengard B, Feng S, Alfrey E, Zaroff J, Emond J, Delmonico F, et al. Report of the Crystal City meeting to maximize the use of organs recovered from the cadaver donor. Am J Transplant 2002; 2 (8): 701-11.

7. Arns W, Citterio F, Campistol JM. 'Old-for-old'-new strategies for renal transplantation. Nephrol Dial Transplant 2007; 22 (2): 336-41.

8. Poblete H. XXXIV Cuenta de hemodiálisis crónica en Chile. Obtenido de http://fmc-ag.cl/_file/file_54_cuenta\%20hemodialisis\%202015.pdf [Consultado el 27 de abril de 2015].

9. Jha V, García-García G, Iseki K, Li Z, Naicker S, Yang $\mathrm{CW}$, et al. Chronic kidney disease: global dimension and perspectives. Lancet 2013; 382 (9888): 260-72.

10. Wolfe R, Ashby V, Milford E, Ojo A, Ettenger R, Port F, et al. Comparison of mortality in all patients on dialysis, patients on dialysis awaiting transplantation, and recipients of a first cadaveric transplant. N Engl J Med 1999; 341 (23): 1725-30.

11. Jofré R, López-Gómez J, Moreno F, Sanz-Guajardo D, Valderrábano F. Changes in quality of life after renal transplantation. Am J Kidney Dis 1998; 32 (1): 93-100.
12. Laupacis A, Keown P, Pus N, Krueger H, Ferguson B, Muirhead N, et al. A study of the quality of life and cost-utility of renal transplantation. Kidney int 1996; 50 (1): 235-42.

13. Randhawa PS, Minervini MI, Lombardero M, Duquesnoy R, Fung J, Demetris A, et al. Biopsy of marginal donor kidneys: correlation of histologic findings with graft dysfunction. Transplantation 2000; 69 (7): 1352 7.

14. Moers C, Smits JM, Maathuis MH, Treckmann J, van Gelder F, Ploeq RJ, et al. Machine perfusion or cold storage in deceased-donor kidney transplantation. $\mathrm{N}$ Engl J Med 2009; 360 (1): 7-19.

15. Port FK, Bragg-Gresham JL, Metzger RA, Dykstra DM, Gillespie BW, Held PJ, et al. Donor characteristics associated with reduced graft survival: an approach to expanding the pool of kidney donors. Transplantation 2002; 74 (9): 1281-6.

16. Remuzzi G, Cravedi P, Perna A, Dimitrov B, Turturro $\mathrm{M}$, Ruggenenti $\mathrm{P}$, et al. Long-Term Outcome of Renal Transplantation from Older Donors. N Engl J Med 2006; 354 (4): 343-52.

17. Pascual J, Zamora J, Pirsch J. A systematic review of kidney transplantation from expanded criteria donors. Am J Kidney Dis 2008; 52 (3): 553-86.

18. Rao P, Merion R, Ashby V, Port F, Wolfe R, Kayler L. Renal transplantation in elderly patients older than 70 years of age: results from the Scientific Registry of Transplant Recipients. Transplantation 2007; 83 (8): 1069-74.

19. Bentas W, Jones J, Karaoguz A, Tilp U, Probst M, Gossmann J, et al. Renal transplantation in the elderly: surgical complications and outcome with special emphasis on the Eurotransplant Senior Programme. Nephrol Dial Transplant 2008; 23 (6): 2043-51.

20. Barba J, Zudaire JJ, Robles JE, Rosell D, Berian JM, Pascual I. Complications of kidney transplantation with grafts from expanded criteria donors. World J Urol 2013; 31 (4): 893-900.

21. Giessing M, Budde K, Fritsche L, Slowinski T, Tuerk I, Loening SA, et al. "Old-for-old" cadaveric renal transplantation: surgical findings, perioperative complications and outcome. Eur Urol 2003; 44 (6): 701-8.

22. Hernández D, Rufino M, Armas S, González A, Gutiérrez $\mathrm{P}$, Torres A, et al. Retrospective analysis of surgical complications following cadaveric kidney transplantation in the modern transplant era. Nephrol Dial Transplant 2006; 21 (10): 2908-15. 